\title{
First report of rapid, non-invasive, and reagent-free detection of malaria through the skin of patients with a beam of infrared light
}

\section{Gabriela Garcia}

Laboratório de Transmissores de Hematozoários, Instituto Oswaldo Cruz, Fiocruz, Rio de Janeiro

Tharanga Kariyawasam

School of Biological Sciences, Faculty of Science, The University of Queensland, Brisbane

Anton Lord

Spectroscopy and Data Consultants Pty Ltd

Cristiano Costa

Fundação de Vigilância em Saúde, Manaus

\section{Lana Chaves}

Laboratório de Imunoparasitologia, Instituto Oswaldo Cruz, Fiocruz, Rio de Janeiro

Josue Lima-Junior

Laboratório de Imunoparasitologia, Instituto Oswaldo Cruz, Fiocruz, Rio de Janeiro

Rafael Maciel-de-Freitas

Laboratório de Transmissores de Hematozoários, Instituto Oswaldo Cruz, Fiocruz, Rio de Janeiro

Maggy Sikulu-Lord ( $\sim$ maggy.lord@uq.edu.au)

School of Biological Sciences, Faculty of Science, The University of Queensland, Brisbane

\section{Research Article}

Keywords:

Posted Date: February 2nd, 2022

DOI: https://doi.org/10.21203/rs.3.rs-1179114/v2

License: (1) (1) This work is licensed under a Creative Commons Attribution 4.0 International License.

Read Full License 
1 First report of rapid, non-invasive, and reagent-free detection of malaria through the skin of 2 patients with a beam of infrared light

3 Gabriela A. Garcia ${ }^{1}$, Tharanga N. Kariyawasam ${ }^{2}$, Anton R Lord ${ }^{3}$, Cristiano Fernandes da Costa ${ }^{4}$ 4 Lana Bitencourt Chaves ${ }^{5}$, Josué da Costa Lima-Junior ${ }^{5}$, Rafael Freitas ${ }^{1,6^{*}}$, Maggy T. Sikulu5 Lord $^{2 *}$

6 'Laboratório de Transmissores de Hematozoários, Instituto Oswaldo Cruz, Fiocruz, Rio de Janeiro, 7 Brazil.

$8{ }^{2}$ School of Biological Sciences, Faculty of Science, The University of Queensland, Brisbane, 9 Australia

$10 \quad{ }^{3}$ Spectroscopy and Data Consultants Pty Ltd

11 Fundação de Vigilância em Saúde, Manaus, Brazil.

$12{ }^{5}$ Laboratório de Imunoparasitologia, Instituto Oswaldo Cruz, Fiocruz, Rio de Janeiro, Brazil.

$13{ }^{6}$ Instituto Nacional de Ciência e Tecnologia em Entomologia Molecular, Universidade Federal do 14 Rio de Janeiro, UFRJ, Brazil.

$15 *$ Equal contribution 


\section{Abstract}

19 We describe the first application of the Near-infrared spectroscopy (NIRS) technique to detect

20 Plasmodium falciparum and $P$. vivax malaria parasites through the skin of malaria positive and

21 negative human subjects. NIRS is a rapid, non-invasive and reagent free technique which

22 involves rapid interaction of a beam of light with a biological sample to produce diagnostic

23 signatures in seconds. We used a handheld, miniaturized spectrometer to shine NIRS light on the

24 ear, arm and finger of $P$. falciparum $(n=7)$ and $P$. vivax $(n=20)$ positive people and malaria

25 negative individuals $(n=33)$ in a malaria endemic setting in Brazil. Supervised machine learning

26 algorithms for predicting the presence or absence of malaria were applied to predict malaria

27 infection status in independent individuals $(\mathrm{n}=12)$. Separate machine learning algorithms for

28 differentiating $P$. falciparum from $P$. vivax infected subjects were developed using spectra from

29 the arm and ear of $P$. falciparum and $P$. vivax $(\mathrm{n}=108)$ and the resultant model predicted

30 infection in spectra of their fingers $(n=54)$.

31 NIRS non-invasively detected malaria positive and negative individuals that were excluded from

32 the model with $100 \%$ sensitivity, $83 \%$ specificity and $92 \%$ accuracy $(n=12)$ with spectra

33 collected from the arm. Moreover, NIRS also correctly differentiated $P$. vivax from $P$.

34 falciparum positive individuals with a predictive accuracy of $93 \%(n=54)$.

35 These findings are promising but further work on a larger scale is needed to address several gaps

36 in knowledge and establish the full capacity of NIRS as a non-invasive diagnostic tool for

37 malaria. It is recommended that the tool is further evaluated in multiple epidemiological and

38 demographic settings where other factors such as age, mixed infection and skin colour can be

39 incorporated into predictive algorithms to produce more robust models for universal diagnosis of 40 malaria. 
44 In 2020, an estimated 241 million malaria-related cases and 627,000 malaria-related deaths were 45 reported by WHO [1]. The Plasmodium parasites which cause the disease are transmitted to 46 people by bites of infected female Anopheles mosquitoes. Among them, the two major

47 Plasmodium parasite species are $P$. falciparum and $P$. vivax. In 2020, $P$. falciparum accounted 48 for $98 \%$ of estimated malaria cases globally and $99.7 \%$ of the cases in the WHO African region. 49 Plasmodium vivax is the predominant parasite in the WHO Region of the Americas, representing $5068 \%$ of malaria cases in the region [1] . In 2015, WHO set a strategy to guide countries towards 51 malaria elimination. The main goal of this strategy is to reduce global malaria incidences and 52 mortality by at least $90 \%$ and eliminate malaria in at least 35 countries by the year 2030 [2]. One 53 of the pillars on which this strategy is based on is to ensure universal access to malaria 54 prevention, diagnosis and treatment. To achieve this target, WHO recommends universal 55 diagnostic testing to all suspected cases. This is particularly crucial in endemic areas where a 56 majority of the malaria infected population has been reported to be asymptomatic [3] as well as 57 in low malaria transmission settings where the proportion of asymptomatic population among the 58 infected individuals can be as high as 60\% [4]. Universal diagnosis is expected to prompt and 59 facilitate the treatment of asymptomatic carriers and limit further community transmission.

60 Optical microscopy, rapid diagnostic tests and molecular tests are the three main diagnostic 61 techniques currently available in malaria endemic regions for malaria diagnosis. Each of these 62 techniques has its own advantages and limitations. Microscopy is the traditional way of detecting 63 malaria parasites in stained thick or thin peripheral blood films using Giemsa, Wrights or Fields

64 stains. Thick film blood films are used to detect the presence of malaria parasite whereas thin 65 blood films are often used to confirm the Plasmodium species [5]. It is the most widely used 66 technique for malaria diagnosis due to its low cost, simplicity and its capacity to detect parasites,

67 differentiate Plasmodium species and estimate the parasite concentration. However, microscopy

68 is technically demanding, time-consuming and requires specialized expertise to accurately

69 identify parasites and differentiate species in samples with low parasitaemia or samples with 70 mixed infections [6-8]. As the average microscopist detection limits are estimated to 50-100 71 parasites $/ \mu \mathrm{L}$, the likelihood of underestimating infection rates particularly in low transmission 72 settings or among asymptomatic population where parasitaemia is low has been reported [9]. 
73 Microscopy is also often unavailable in rural settings where power supply can be problematic 74 [10].

75 Rapid Diagnostic tests (RDTs) are designed to detect malaria antigens in blood by targeting 76 falciparum-specific protein such as histidine-rich protein II (HRP-II) or lactate dehydrogenase 77 (LDH) [11, 12]. RDTs are simple, relatively cheap and can be used in remote areas without 78 specialized equipment or need for electricity (Murray et al., 2003). However, like microscopy, 79 RDTs can only reliably detect 50-100 parasites/ $\mu \mathrm{L}[11,12]$, they have to be coupled with other 80 techniques in areas with more than one malaria parasite species due to their inability to 81 differentiate Plasmodium species $[9,13]$ and they can generate false positives due to the 82 persistence of HRP- II following an infection [14].

83 Molecular tests such as Polymerase chain reaction (PCR) are currently the most accurate and the 84 most sensitive techniques for detecting malaria in low or submicroscopic samples, for mixed 85 infections and for differentiating Plasmodium species [15-17]. However, molecular techniques 86 are feasible diagnostic tools for universal diagnosis due to the high cost, time and skills required 87 to operate them [18].

88 Here we explored a novel technique for large scale and universal diagnosis of malaria that could 89 potentially be scaled up as a complementary tool to guide the proposed malaria elimination 90 strategy. The near-infrared spectroscopy (NIRS) is a simple, non-invasive, reagent free technique 91 that uses the near-infrared light to characterize biological samples. The technique involves 92 shining a beam of light on a sample for approximately 5 seconds and subsequent collection of a 93 spectral signature. The spectral signature is a reflection of the chemical composition of a sample 94 and can be analyzed using machine learning techniques to determine its diagnostic features.

95 Here, we used a handheld NIRS spectrometer to non-invasively collect spectral signatures from 96 ears, arms and fingers of malaria positive and negative individuals living in a highly endemic 97 malaria transmission area in Brazil where both $P$. falciparum and $P$. vivax is prevalent at a $9830 / 70 \%$ ratio historical proportion. This provided us an opportunity to test the capacity of NIRS 99 albeit on a small scale, to non-invasively differentiate malaria positive and negative individuals 100 and to differentiate those infected with P. falciparum from those with $P$. vivax. 


\section{Study area, population and malaria prevalance}

104 The study was conducted in the municipality of São Gabriel da Cachoeira (SGC), located in the 105 Upper Rio Negro region, State of Amazonas, Northern Brazil (007’51'S, 6705'15’W). SCG is 106 surrounded by the Amazon and is the municipality with the highest percentage of self-declared 107 indigenous people (over 75\%) in Brazil, with an estimated 30,000 people living in the area, and 108 over 20 ethnic groups [19]. This area represents $99.8 \%$ of all malaria cases in Brazil and records 109 both $P$. vivax and $P$. falciparum. In 2019, a total of 8,605 autochthonous cases were reported in 110 SCG. From January 2019 to June 2020 an estimated 34\% of malaria cases were caused by $P$. 111 falciparum and mixed infections [20].

\section{Ethics approval}

114 The study was approved by the Instituto Oswaldo Cruz-Fiocruz human ethics committee (Ethics 115 protocol No. 94070418.7.0000.5248) and all methods described here were performed in 116 accordance with the relevant guidelines and regulations. Patients with malaria symptoms that 117 were seeking diagnosis and treatment at the basic health units were approached to volunteer for 118 this study. Prior to recruitment, a written informed consent was obtained from patients after the 119 nature and possible consequences of the studies were explained. The written consent form was 120 previously approved by the institutes Ethical committee. Further work to determine presence of 121 malaria parasites in volunteer's blood was approved by the National Genetic Heritage 122 Management System (A88B262).

NIRS instrument used

125 NIRvascan Near infrared spectrometer reflective model G1 (Allied scientific pro) was used in 126 this study. The model used is a diffuse reflectance spectrometer with wavelength ranging from 127 900-1700nm, a 5000:1 signal to noise ratio for 1 second and an optical resolution of 10nm pixel 
resolution. It has an inGaAs detector (Hamamatsu model G12180-010A), a dark current of 0.8nA

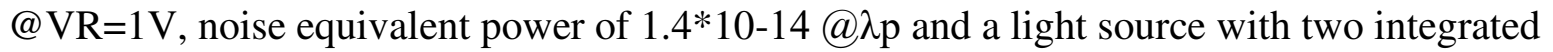
tungsten halogen lamps with 1 watt and built in at 45 degrees from the surface. It weighs $136 \mathrm{~g}$ and measures $82.2 \times 63 \times 40 \mathrm{~mm}$, rechargeable and can be operated by either a computer or a 132 smartphone via Bluetooth.

\section{Scanning}

134 Participants presenting with malaria symptoms were scanned with the NIRvascan spectrometer 135 which was connected to a notebook using Bluetooth. Once connected, the participants arm, ear 136 and finger were one after the other placed directly onto the spectrometer's scan window and 137 spectra was collected by pressing the scan button (Fig 1A). Two spectra were collected from 138 each body part scanned for each patient. A total of 60 patients were scanned and a total of 360 139 spectra were collected. An example of the average raw spectra collected from each body part for 140 malaria positive and malaria negative individuals is shown in Fig 1B and the average raw spectra 141 collected from $P$. falciparum and $P$. vivax infected individuals is shown in Fig 1C.

Fig 1
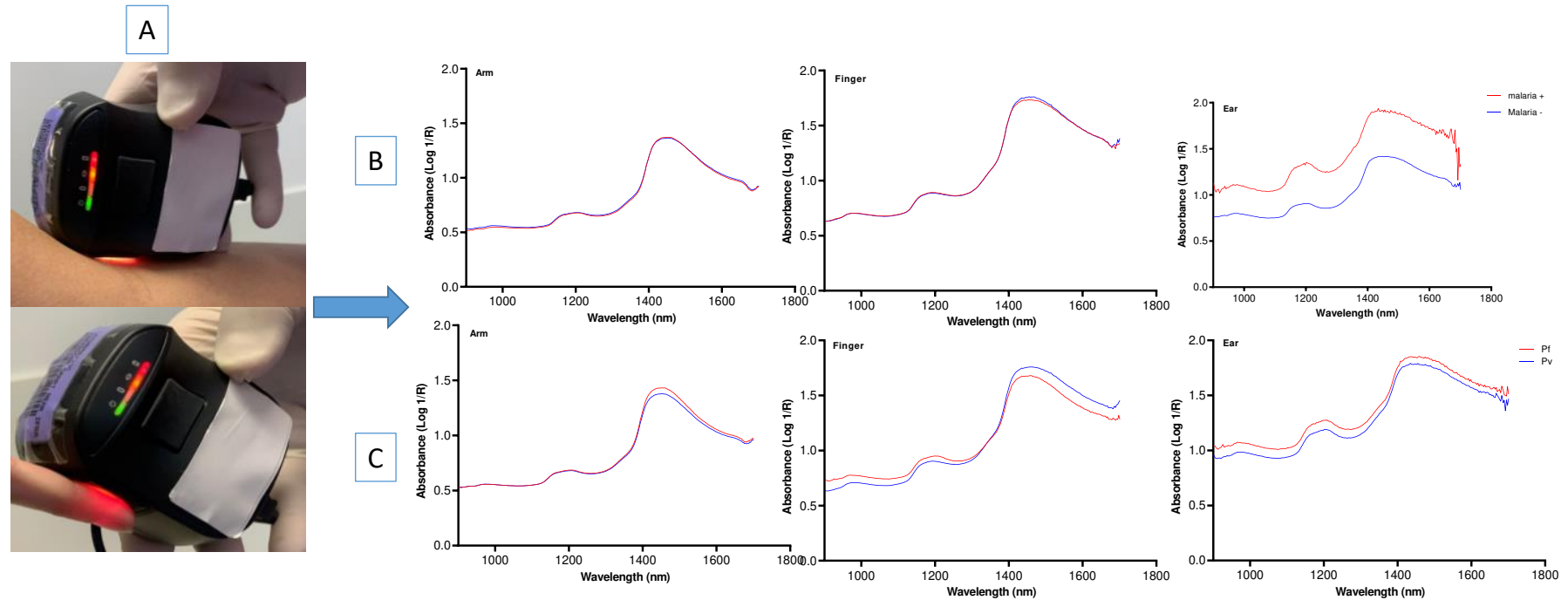

143 Fig 1. Shows non-invasive scanning of the arm and the finger (panel A), raw spectra collected

144 from the ear, arm and finger for malaria infected and non-infected individuals (Panel B) and spectra for $P$. falciparum and $P$. vivax infected individuals (Panel C) 
147 PCR confirmation of positive and negative cases

148 Genomic DNA from whole blood samples was extracted using the QIAamp DNA Blood Mini Kit 149 according to the manufacturer's instructions (Qiagen, Hilden, Germany) and stored at $-20^{\circ} \mathrm{C}$ until

150 amplification. Detection and identification of Plasmodium was performed using Nested PCR with 151 specific primers for genus (Plasmodium sp.) and species (P. falciparum and P. vivax) as described 152 by Snounou et al. [21, 22]. In the first amplification reaction, $3 \mu \mathrm{L}$ of purified genomic DNA were 153 used in a $25 \mu \mathrm{L}$ reaction with genus specific primers. During the second PCR reaction, $3 \mu \mathrm{L}$ of 154 PCR amplification product of the first reaction was used as a template in a $25 \mu \mathrm{L}$ reaction with 155 species specific primers. The amplified PCR products were size-fractionated by electrophoresis in $1562 \%$ agarose gel (Sigma Aldrich, Missouri, USA), 1× TAE buffer (0.04 M TRIS-acetate, $1 \mathrm{mM}$ 157 EDTA) in the presence of $1 \times$ GelRed nucleic acid stain (Biotium, Fremont, CA, USA). PCR 158 products were visualized by ultraviolet (UV) illumination. The presence or absence of Plasmodium 159 species was determined by species-specific amplicon sizes.

160 Data analysis

161 Spectral signatures were organized in MS Excel spreadsheet before they were imported to JMP 162 pro 16.2 for analysis using JMP version 16 software (SAS institute, North Carolina, United States). 163 Data in excel spreadsheet was classified according to patient ID, age, skin color, gender, height, 164 infection status and species identity.

165 In JMP, supervised machine learning was used to analyze the data. The first supervised learning 166 involved pooling all data collected from infected patients regardless of the Plasmodium parasite 167 they were infected with. This allowed us to differentiate malaria infected from uninfected patients. 168 Spectra was divided into a training set (60\% with 216 spectral signatures from 36 patients), a 169 validation set (20\% with 72 spectral signatures from 12 subjects) and a test set (20\% with 72 170 spectral signatures from 12 subjects). We applied the model screening feature to simultaneously 171 fit several machine learning algorithms on the data to allow us to compare and select the best 172 predictive model for infection prediction. Malaria infection status was used as the response factor 173 and the spectral signatures from 900-1700nm, colour skin, age and gender were used as predictors. 
174 The best predictive model selected for differentiating infected from uninfected samples was 175 bootstrap forest with 100 trees. The minimum splits per tree was 10 and the minimum size split 176 was 5.

177 The second supervised learning was used to develop models for differentiating $P$. vivax from $P$. 178 falciparum infected people. Due to a very low number of $P$. falciparum infected patients i.e. 7 179 compared to 20 infected with $P$. vivax, the data was split into a training set and a validation set. 180 The training set consisted of all spectral signatures collected from the ears and arms of all patients 181 (66\% of the data with 108 spectral signatures) whereas data collected from the fingers (33\% of the 182 data with 54 spectral signatures) was used as a validation set. Similarly, we applied the model 183 screening feature to simultaneously fit several machine algorithms on the data to allow us to 184 compare and select the best predictive model for differentiating $P$. falciparum infected from $P$. 185 vivax infected patients. Plasmodium species type was selected as the response factor and spectral 186 signatures, age, gender and skin colour were used as predictors. The best predictive model selected 187 for differentiating infected from uninfected samples was boosted tree.

188 For both data sets, a second order Savitzky-Golay derivative with 15 smoothing points was applied to all raw spectra to allow visualization of peaks of importance. The summary of all results 190 is presented as an average of two spectra collected/body part. An individual was considered 191 infected if at least one of the spectrum collected was predicted as infected and an individual was considered negative if both spectra were predicted as negative.

\section{Results}

\section{Differentiating infected from uninfected patients}

195 PCR confirmed 45\% ( $=27)$ of the 60 people scanned were positive with malaria while the rest were malaria negative. Of the malaria positive individuals, $75 \%(n=20)$ and $25 \%(n=7)$, were 197 infected with $P$. vivax and P. falciparum, respectively.

198 Absorption differences were seen from the raw spectra collected from malaria positive and malaria 199 negative patients. Malaria positive patients were generally seen to have higher absorbance values 200 than malaria negative patients (Fig 1B). There were also clear differences between infected and 201 uninfected patients from the second derivative spectra (Fig 2). Distinct absorption bands involved 
202 in the differentiation of malaria positive and malaria negative patients were observed within the $2031^{\text {st }}$ and $2^{\text {nd }}$ overtone regions and these bands were present in at least two body parts scanned. They 204 include absorption bands around 1120, 1160, 1337, 1370, 1408, 1626 and $1661 \mathrm{~nm}$ (Fig 2). Among 205 them, those that were reduced in malaria infected patients include bands around 1160, 1408, and $2061661 \mathrm{~nm}$ whereas absorption bands that increased in malaria infected patients include those around 2071120,1370 and $1626 \mathrm{~nm}$. Bands in the $2^{\text {nd }}$ region are dominated by overtones of O-H, N-H and $208 \mathrm{C}=\mathrm{H}$ combinations whereas the absorption bands around 1626, 1661 and 1696nm in the first 209 overtone region are dominated by $\mathrm{C}=\mathrm{H}$ and $\mathrm{O}-\mathrm{H}$ compounds related to parasite proteins.

210 The sensitivity and specificity of NIRS and machine learning for differentiating malaria positive 211 from malaria negative patients that were used to test the accuracy of the bootstrap forest model 212 developed is shown in Table 1. Using spectral signatures collected from just the arms, NIRS was $213100 \%$ sensitive, $(n=6), 83 \%$ specific $(n=6)$ and $92 \%$ accurate $(n=12)$ regardless of the Plasmodium 214 species they were infected with. Spectra collected from fingers was $100 \%$ accurate for detecting 215 malaria negative people whereas a combined spectra from fingers/ears and combined spectra from 216 all the three body parts were $83 \%$ sensitive, $100 \%$ specific and $92 \%$ accurate for predicting the 217 presence or absence of malaria parasites.

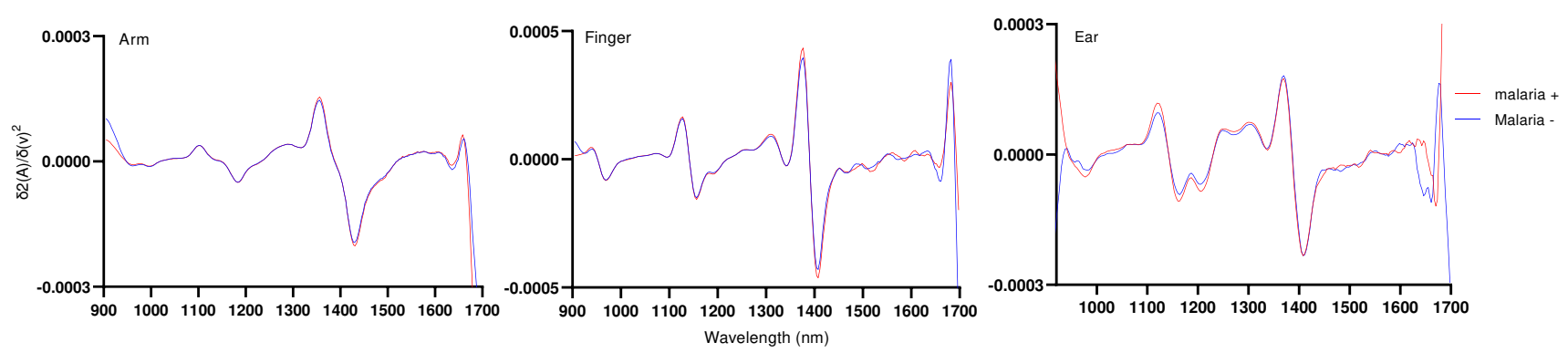

220 Fig 2. Second derivative spectra of malaria infected and uninfected patients for the arm, finger and 221 ear showing absorption peaks of importance 
225 Table 1. Sensitivity, specificity positive predictive rate (PPR), Negative predictive rate (NPR) for 226 malaria positive and negative samples that were used to test the predictive model developed

227

\begin{tabular}{|c|c|c|c|c|c|}
\hline $\begin{array}{l}\text { Body part } \\
\text { scanned }\end{array}$ & $\begin{array}{l}\% \text { Sensitivity } \\
(\mathrm{N}=6)\end{array}$ & $\begin{array}{l}\text { \%Specificity } \\
{[\mathrm{N}=6]}\end{array}$ & $\begin{array}{l}\% \mathrm{PPR} \\
{[\mathrm{N}=12]}\end{array}$ & $\begin{array}{l}\% \mathrm{NPR} \\
{[\mathrm{N}=12]}\end{array}$ & $\begin{array}{l}\% \text { Accura } \\
\text { cy } \\
{[\mathrm{N}=12]}\end{array}$ \\
\hline Arm & 100 & 83 & 86 & 100 & 92 \\
\hline Ear & 66 & 50 & 75 & 66 & 58 \\
\hline Finger & 66 & 100 & 100 & 75 & 83 \\
\hline $\begin{array}{l}\text { Arm and } \\
\text { Finger }\end{array}$ & 83 & 83 & 75 & 75 & 83 \\
\hline $\begin{array}{l}\text { Arm and } \\
\text { Ear }\end{array}$ & 83 & 83 & 75 & 75 & 83 \\
\hline $\begin{array}{l}\text { Finger and } \\
\text { ear }\end{array}$ & 83 & 100 & 100 & 86 & 92 \\
\hline $\begin{array}{l}\text { Arm, Ear } \\
\text { and Finger }\end{array}$ & 83 & 100 & 100 & 86 & 92 \\
\hline
\end{tabular}




\section{Hemozoin and hemoglobin absorption bands relative to published work}

235 When Plasmodium parasites consume hemoglobin in red blood cells they produce a toxic 236 compound known as heme. Heme is detoxified by the parasite into hemozoin pigment through 237 metabolisation of hemoglobin. Hemozoin is therefore considered a powerful and reliable 238 biomarker for malaria infection. Using a second derivative average spectra of malaria positive and 239 negative patients, we observed several bands that have previously been reported as hemozoin 240 absorption bands. Fig 3 shows absorption bands around 1451, 1495 and 1624nm that were also 241 observed from dry crystalline hemozoin, bands around 1505, 1550 and 1642 observed from 242 synthetic hemozoin ( $\beta$-hematin) and bands around $1515 \mathrm{~nm}$ observed from dry hemozoin isolated 243 from infected red blood cells[23]. Absorption bands around 1642 and 1695nm in the second 244 overtone region are related to hemoglobin as earlier reported by Kuenstner and Norris [24] and 245 more recently by Adegoke and colleagues [23]. Both of these bands were reduced in malaria 246 infected patients. A reduction in hemoglobin concentration is expected for a malaria infected 247 patient as the parasite consumes hemoglobin and other red blood cells proteins to produce 248 hemozoin. 


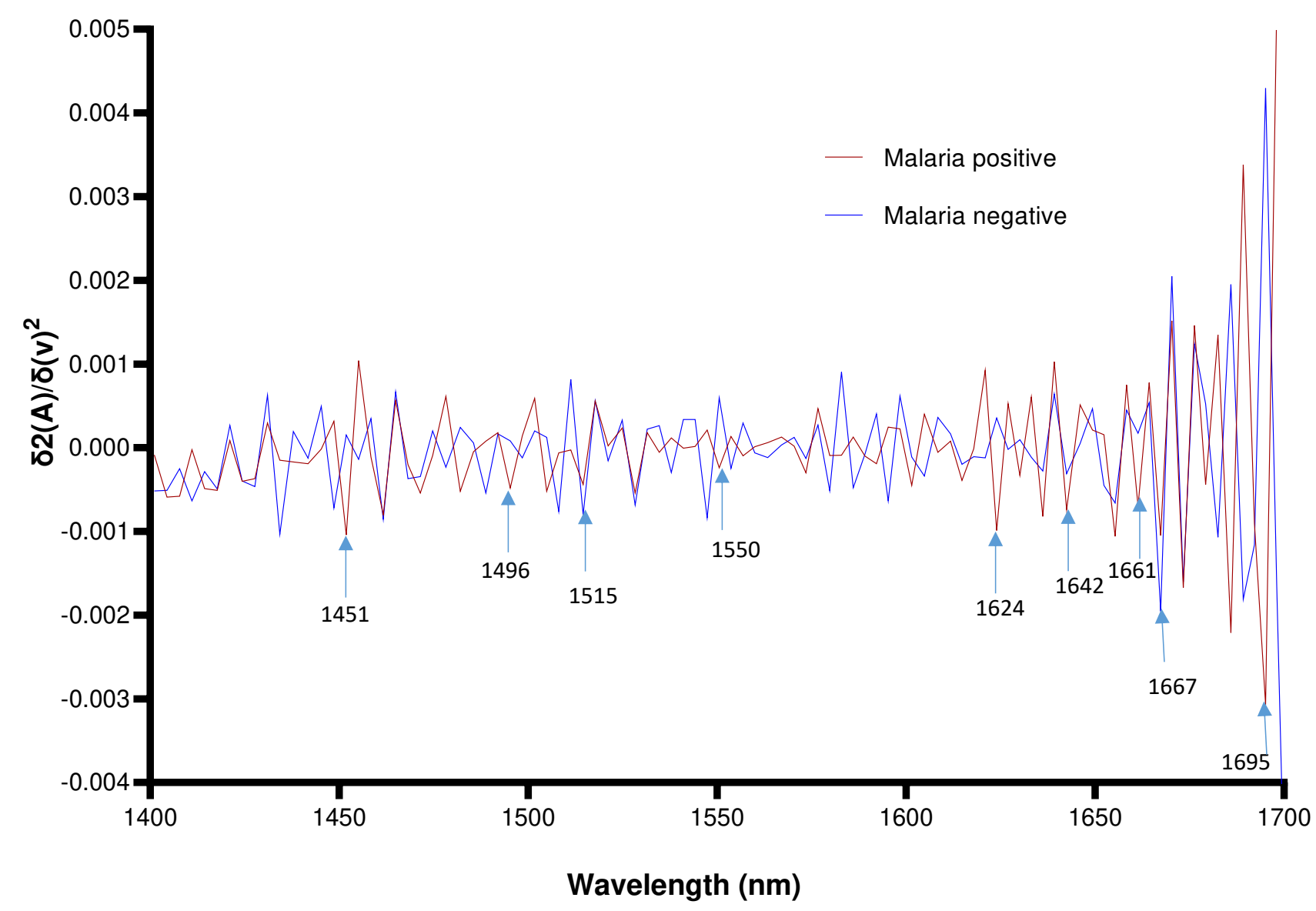

250 Fig 3. Second derivative spectra of malaria positive and negative patients showing different 251 absorption peaks of hemozoin and hemoglobin proteins that have previously been reported

\section{NIRS differentiation of Plasmodium falciparum and vivax}

253 The average raw spectra (Fig 1C) and the second derivative spectra (Fig 4A) of P. falciparum and $254 P$. vivax infected patients from the ear, arm and the finger indicates that across the entire spectrum, 255 patients with $P$. falciparum parasites generally absorbed more light compared to those infected 256 with $P$. vivax. Differences between the two malaria species were observed at absorption bands 257 around 960, 1171, 1412 and 1530nm. However major absorption differences dominated within the 258 1400-1600nm spectral region (Fig 4B).

259 Using boosted tree supervised machine learning, the model that was developed using spectral 260 signatures collected from the ears and arms of $P$. falciparum and $P$. vivax positive patients was 
261100 percent accurate for differentiating patients into these two groups. When the model was used 262 to predict infection using spectra signatures collected from fingers (i.e. the validation set) that were 263 excluded from the model, only one patient from each group was misclassified $(\mathrm{N}=27)$. The 264 sensitivity for detecting $P$. falciparum and $P$. vivax was $86 \%(\mathrm{~N}=7)$ and $95 \%(\mathrm{~N}=20)$, respectively 265 (Fig 4C).

Fig 4

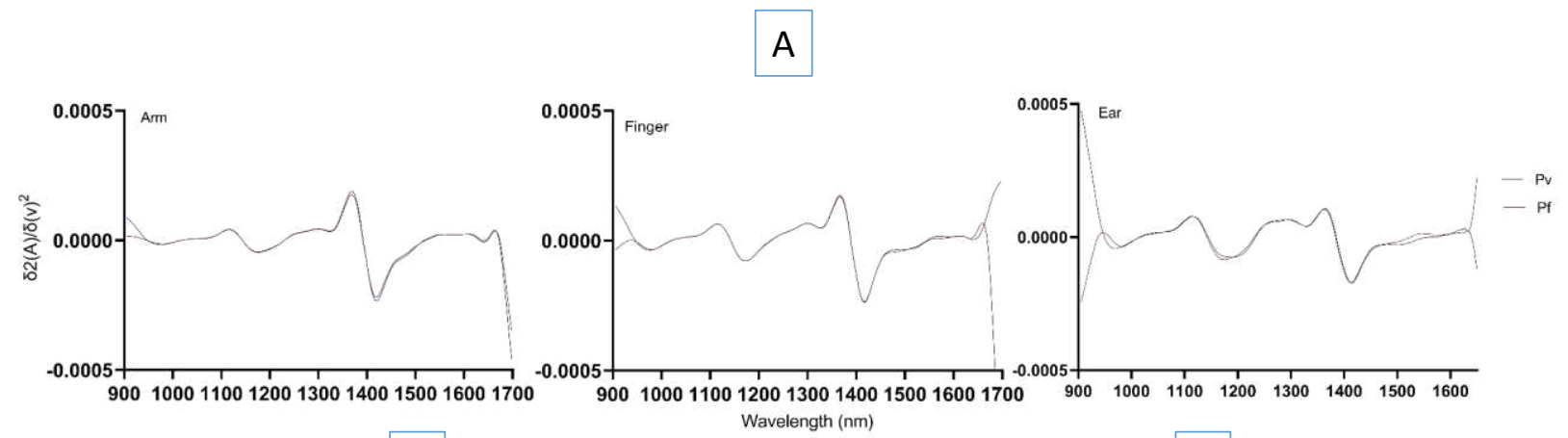

B

266

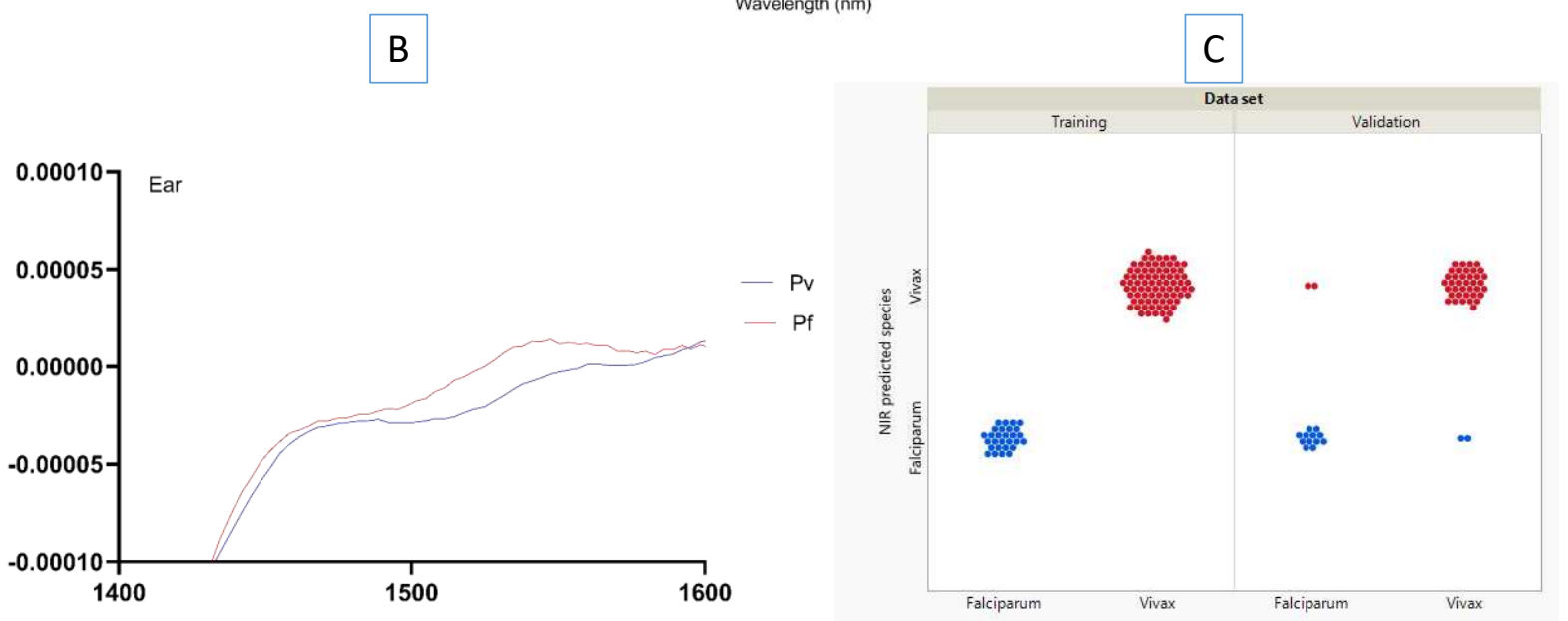

267 Fig 4. Second derivative spectra of $P$. falciparum and $P$. vivax from the arm, finger and ear (Panel 268 A), enlarged region showing major NIRS absorption differences between patients infected with $P$. 269 falciparum and patients with $P$. vivax (Panel B) and summary of the sensitivity of NIRS for 270 differentiating P. falciparum from P. infected positive patients (Panel C). 
274 We assessed if a non-invasive and reagent-free technique that uses the NIR region of the 275 electromagnetic spectrum can detect and differentiate $P$. falciparum and $P$. vivax parasite through 276 the skin of positive and negative patients in Amazon, Brazil. We hypothesized that the invasion of 277 Plasmodium parasites into the human host's red blood cells results in significant structural, 278 biochemical and functional changes which are expected to generate a unique spectrum for malaria 279 positive and negative individuals. They include the loss of the discoid shape of the red blood cells, 280 reduced hemoglobin concentration, increased adhesiveness and permeability to Plasmodium species infection [25]. In addition, over 400 Plasmodium parasite related proteins including the commonly used biomarker for malaria infection i.e. the hemozoin protein, hydroxy fatty acids and lipids are released into red cells [26-28] which are also expected to absorb light at specific wavelengths to generate unique absorption bands (Fig 4).

We also tested the hypothesis that $P$. vivax and $P$. falciparum cause remarkable differences to host cells to generate a unique NIR spectrum. For example, it has been reported that an infection with $P$. vivax results into parasitised red cells that are enlarged, pale, fine stippling known as Schuffener's dots [29] whereas infection with $P$. falciparum results in fine stippling non-enlarged parasitised cells known as Maurer's clefts [30]. There are also remarkable genomic and structural differences between schizonts, trophozoites and gametocytes of the two species that are expected to generate a unique spectral signature [31].

292 Our preliminary findings using 27 malaria positive and 33 malaria negative individuals have 293 confirmed the two hypotheses. The spectral signatures collected from malaria positive individuals 294 regardless of whether they were positive with $P$. falciparum or $P$. vivax were seen to produce 295 absorption peaks that were significantly different between malaria positive and negative 296 individuals. Similarly, remarkable differences were observed for spectral signatures of $P$. 297 falciparum positive and $P$. vivax indicating their chemical profile was indeed different. The 298 absorption peaks identified in this study are consistent with previous findings that also used NIRS 299 to detect $P$. falciparum and hemoglobin in red blood cells in vitro [23, 24]. However, this is the 300 first study to demonstrate that NIRS spectral signatures obtained non-invasively through the skin 301 of human subjects carry diagnostic features for malaria. Findings from previous studies indicated 
302 that the technique can also non-invasively detect various infections in heads, thoraces and 303 abdomens of mosquitoes such as malaria [32], Zika [33], Wolbachia [34] and Chikungunya [35] 304 and more recently to detect Chagas disease in Triatomine bugs. NIRS has also been used 305 extensively to detect cancer tumors in patients [36-38].

306 Current malaria elimination strategies require high throughput and point of care diagnostic 307 approaches that can facilitate timely diagnosis and identify asymptomatic carriers. Although on a 308 small scale, this preliminary study provides prior evidence to support the potential use of a cheap, 309 battery-operated, portable infrared spectrometer for this purpose. By removing the need to draw 310 blood, non-invasive diagnosis of malaria by NIRS has the potential to revolutionize our ability to 311 rapidly detect malaria in human populations. Non-invasive scanning will also provide a justifiable, 312 affordable and rapid platform for screening malaria in asymptomatic populations and make malaria 313 diagnosis among babies a painless undertaking. Following the development of diagnostic models 314 using machine learning techniques, NIRS could enable thousands of samples/individuals to be 315 screened in a day with unskilled personnel. With a single scan, samples could easily be 316 differentiated into infection status and parasite species therefore significantly reducing the time 317 and cost required to produce two individual tests. Furthermore, predictive algorithms could be 318 incorporated into a cloud-based system for real time point of care diagnosis and real time 319 monitoring of malaria infections on a large-scale and multiple locations including rural areas. 320 Cloud-based and real time diagnosis means data will be available to decision makers in real time 321 thereby facilitating rapid decision making and timely distribution of resources where required to 322 stop outbreaks. NIRS is a unique tool that could facilitate timely isolation and treatments of 323 infected cases, through mass screening of population at risk including at ports of entry such as 324 airports or even at household levels to stop transmission to local mosquito populations by overseas 325 travelers, reduce global outbreaks and prevent re-introduction in areas under the elimination phase. 326 The NIRS unit (Fig 1) that was assessed under this study is an off-the-shelf spectrometer that could 327 easily be integrated and scaled up into existing programmatic malaria evaluations. However, 328 further work using a larger sample size from multiple malaria epidemiological settings is required 329 to develop robust predictive models incorporating different demographics including malaria 330 species, parasitaemia level, clinical status, age groups, blood groups, skin color and most 331 importantly mixed/co-infections of malaria or other pathogens such as soil transmitted parasites. 332 Future work should also assess the capacity of NIRS to quantify parasitaemia of infected 
333 individuals. Although our results are based on a limited sample size and remain inconclusive at 334 this stage, nevertheless our findings demonstrate the potential of NIRS to non-invasively diagnose 335 malaria in infected people and to differentiate P. falciparum from P. vivax infected individuals. 336 The study also represents initial steps towards development of the first non-invasive, light-based 337 technique for other blood-borne viruses and parasites. 
344 1. WHO, World malaria report. World Health Organisation, 2021.

345 2. Organization, W.H., Global technical strategy for malaria 2016-2030. 2015: World Health

3. Lin, J.T., D.L. Saunders, and S.R. Meshnick, The role of submicroscopic parasitemia in malaria transmission: what is the evidence? Trends in parasitology, 2014. 30(4): p. 183190.

4. Sturrock, H.J., et al., Targeting asymptomatic malaria infections: active surveillance in control and elimination. PLoS medicine, 2013. 10(6): p. e1001467.

5. Warhurst, D. and J. Williams, ACP Broadsheet no 148. July 1996. Laboratory diagnosis of malaria. Journal of clinical pathology, 1996. 49(7): p. 533.

6. Mwangi, T.W., et al., Clinical algorithms for malaria diagnosis lack utility among people of different age groups. Tropical Medicine \& International Health, 2005. 10(6): p. 530536.

7. Berzosa, P., et al., Comparison of three diagnostic methods (microscopy, RDT, and $P C R)$ for the detection of malaria parasites in representative samples from Equatorial Guinea. Malaria Journal, 2018. 17(1): p. 333.

8. Payne, D., Use and limitations of light microscopy for diagnosing malaria at the primary health care level. Bulletin of the World Health Organization, 1988. 66(5): p. 621.

9. Moody, A., Rapid diagnostic tests for malaria parasites. Clinical microbiology reviews, 2002. 15(1): p. 66-78.

10. Cunha, C.B. and B.A. Cunha, Brief history of the clinical diagnosis of malaria: from Hippocrates to Osler. J Vector Borne Dis, 2008. 45(3): p. 194-199.

11. Murray, C.K., et al., Rapid diagnostic testing for malaria. Tropical Medicine \& International Health, 2003. 8(10): p. 876-883.

12. Rubio, J., et al., Limited level of accuracy provided by available rapid diagnosis tests for malaria enhances the need for PCR-based reference laboratories. Journal of Clinical Microbiology, 2001. 39(7): p. 2736-2737.

13. McMorrow, M.L., M. Aidoo, and S.P. Kachur, Malaria rapid diagnostic tests in elimination settings — can they find the last parasite? Clinical Microbiology and Infection, 2011. 17(11): p. 1624-1631.

14. Humar, A., et al., ParaSight $\Theta F$ test compared with the polymerase chain reaction and microscopy for the diagnosis of Plasmodium falciparum malaria in travelers. The American journal of tropical medicine and hygiene, 1997. 56(1): p. 44-48.

15. Brown, A.E., et al., Demonstration by the polymerase chain reaction of mixed Plasmodium falciparum and $P$. vivax infections undetected by conventional microscopy. Transactions of the Royal society of tropical medicine and hygiene, 1992. 86(6): p. 609612.

16. Morassin, B., et al., One year's experience with the polymerase chain reaction as a routine method for the diagnosis of imported malaria. The American journal of tropical medicine and hygiene, 2002. 66(5): p. 503-508.

17. Patsoula, E., et al., A single-step, PCR-based method for the detection and differentiation of Plasmodium vivax and P. falciparum. Annals of Tropical Medicine \& Parasitology, 2003. 97(1): p. 15-21.

18. Organization, W.H., New perspectives: malaria diagnosis. Report of a Joint WHO/USAID Informal Consultation, 25-27 October 1999. New perspectives: malaria diagnosis. Report of a Joint WHO/USAID Informal Consultation, 25-27 October 1999., 2000.

19. Oliveira-Ferreira, J., et al., Malaria in Brazil: an overview. Malaria journal, 2010. 9(1): $p$. $1-15$. 
392

393

394

395

396

397

398

399

400

401

402

403

404

405

406

407

408

409

410

411

412

413

414

415

416

417

418

419

420

421

422

423

424

425

426

427

428

429

430

431

432

433

434

435

436

437

438

439

440

20. Epidemiológico, B., Secretaria de Vigilância em Saúde /Ministério da Saúde. 2020. Número Especial Nov. 2020(ISSN 9352-7864).

21. Snounou, G., et al., High sensitivity of detection of human malaria parasites by the use of nested polymerase chain reaction. Mol Biochem Parasitol, 1993. 61(2): p. 315-20.

22. Snounou, G. and B. Singh, Nested PCR analysis of Plasmodium parasites. Methods Mol Med, 2002. 72: p. 189-203.

23. Adegoke, J.A., et al., A Near-Infrared "Matchbox Size" Spectrometer to Detect and Quantify Malaria Parasitemia. Analytical Chemistry, 2021. 93(13): p. 5451-5458.

24. Kuenstner, J.T. and K.H. Norris, Spectrophotometry of human hemoglobin in the near infrared region from 1000 to $2500 \mathrm{~nm}$. Journal of Near Infrared Spectroscopy, 1994. 2(2): p. 59-65.

25. Mohandas, N. and X. An, Malaria and human red blood cells. Medical microbiology and immunology, 2012. 201(4): p. 593-598.

26. Hiller, N.L., et al., A host-targeting signal in virulence proteins reveals a secretome in malarial infection. Science, 2004. 306(5703): p. 1934-1937.

27. Pandey, A.V., et al., Hemozoin formation in malaria: a two-step process involving histidine-rich proteins and lipids. Biochemical and biophysical research communications, 2003. 308(4): p. 736-743.

28. Schwarzer, E., et al., Malaria-parasitized erythrocytes and hemozoin nonenzymatically generate large amounts of hydroxy fatty acids that inhibit monocyte functions. Blood, The Journal of the American Society of Hematology, 2003. 101(2): p. 722-728.

29. Barnwell, J.W., et al., Plasmodium vivax: malarial proteins associated with the membrane-bound caveola-vesicle complexes and cytoplasmic cleft structures of infected erythrocytes. Experimental parasitology, 1990. 70(1): p. 85-99.

30. Lanzer, M., et al., Maurer's clefts: a novel multi-functional organelle in the cytoplasm of Plasmodium falciparum-infected erythrocytes. International journal for parasitology, 2006. 36(1): p. 23-36.

31. Das, A., et al., Plasmodium falciparum and Plasmodium vivax: so similar, yet very different. Parasitology research, 2009. 105(4): p. 1169-1171.

32. Maia, M.F., et al., Detection of Plasmodium falciparum infected Anopheles gambiae using near-infrared spectroscopy. Malaria journal, 2019. 18(1): p. 1-11.

33. Fernandes, J.N., et al., Rapid, noninvasive detection of Zika virus in Aedes aegypti mosquitoes by near-infrared spectroscopy. Science advances, 2018. 4(5): p. eaat0496.

34. Sikulu-Lord, M.T., et al., Rapid and non-destructive detection and identification of two strains of Wolbachia in Aedes aegypti by near-infrared spectroscopy. PLoS neglected tropical diseases, 2016. 10(6): p. e0004759.

35. Santos, L.M., et al., High throughput estimates of Wolbachia, Zika and chikungunya infection in Aedes aegypti by near-infrared spectroscopy to improve arbovirus surveillance. Communications biology, 2021. 4(1): p. 1-9.

36. Xue, Z., S. Zeng, and J. Hao, Non-invasive through-skull brain vascular imaging and small tumor diagnosis based on NIR-II emissive lanthanide nanoprobes beyond 1500 nm. Biomaterials, 2018. 171: p. 153-163.

37. Kondepati, V.R., H.M. Heise, and J. Backhaus, Recent applications of near-infrared spectroscopy in cancer diagnosis and therapy. Analytical and bioanalytical chemistry, 2008. 390(1): p. 125-139.

38. Abe, K., et al., Non-invasive in vivo imaging of near infrared-labeled transferrin in breast cancer cells and tumors using fluorescence lifetime FRET. PloS one, 2013. 8(11): p. e80269. 